\title{
GW23-e2708 INTERRELATIONSHIP BETWEEN METABOLIC SYNDROME AND FRAMINGHAM RISK SCORES IN ASIAN INDIANS WITH ANGIOGRAPHICALLY DOCUMENTED CORONARY ARTERY DISEASE
}

doi:10.1136/heartjnl-2012-302920d.37

Roopali Khanna, Aditya Kapoor, Sudeep Kumar, Satyendra Tewari, Naveen Garg, Pravin K Goel, Roopali Khanna. Department of Cardiology, Sanjay Gandhi Post Graduate Institute of Medical Sciences, Rae Bareli Road, Lucknow 226014, India

Objectives Metabolic syndrome (MS) with its which is a clustering of atherogenic metabolic abnormalities is considered an important determinant of cardiovascular (CV) risk. Framingham risk scores (FRS) have been traditionally used as predictors of the 10-year risk of coronary heart disease categorise patients into low (risk $<10 \%$ ), intermediate (risk 10-20\%) and high risk groups (>20\%). However FRS often underestimates the CV risk in Asians, younger patients and those with MS. Since Asian Indians often develop coronary artery disease $(\mathrm{CAD})$ at a younger age and also have a high prevalence of MS, more data is needed on the relationship between MS and FRS in the resident Indian population with angiographically documented CAD.

Methods Of 250 patients (age $55.6+7.8$ years) undergoing coronary angiography, Metabolic syndrome was diagnosed in $79 \%$; abdominal obesity, low HDL and hypertension were the commonest of the diagnostic criteria of MS, being present in $>70 \%$ cases. As expected, with progressively increasing BMI, the prevalence of MS increased (36 and 51\% in those with BMI 23-24.9 and BMI $>25 \mathrm{~kg} / \mathrm{m}^{2}$ respectively); however despite having a normal BMI $\left(<23 \mathrm{~kg} / \mathrm{m}^{2}\right), 16 \%$ patients satisfied the diagnostic criteria of MS.

The prevalence of double or triple vessel CAD was significantly more common in patients with MS as compared to those without MS (64 vs 40\%, p=0.02), while the incidence of angiographically normal coronary arteries was also significantly less common in patients with MS as compared to those without MS (8 vs $32 \%$, $\mathrm{p}<0.05)$. Patients with MS had significantly higher mean FRS than those without MS (16.2 vs $8.75, \mathrm{p}<0.0001)$. Most patients with MS (78\%) had an intermediate to high 10 year CV risk (>10\%) as estimated by FRS.

We observed that with increasing FRS risk scores, the proportion of patients having MS also increased, of those with low, intermediate and high FRS, 61, 87 and 92\% respectively had Metabolic Syndrome. To observer the independent effects of MS and FRS, patients were categorised into age quartiles ( $<45$ years, 4555 years, $55-65$ years and $>65$ years). Though the incidence of Metabolic Syndrome was nearly similar amongst all age quartiles (75\%, 79\%, 81\%, 82\% respectively), distribution of FRS among different age groups was quite dissimilar. Despite angiographically documented $\mathrm{CAD}$, in patients $<45$ years none of the patients were categorised as high FRS. Only 15 and $17 \%$ patients could be categorised as having high FRS in age groups $45-55$ and 55-65 years, while in patients older than 65 years, $30 \%$ were classified as having high FRS. This analysis helped highlight the obvious limitation of age dependence of FRS. 
Results Of 250 patients (age 55.6+7.8 years) undergoing coronary angiography, Metabolic syndrome was diagnosed in $79 \%$; abdominal obesity, low HDL and hypertension were the commonest of the diagnostic criteria of $\mathrm{MS}$, being present in $>70 \%$ cases. As expected, with progressively increasing BMI, the prevalence of MS increased (36 and $51 \%$ in those with BMI 23-24.9 and BMI $>25 \mathrm{~kg} / \mathrm{m}^{2}$ respectively); however despite having a normal BMI $\left(<23 \mathrm{~kg} / \mathrm{m}^{2}\right), 16 \%$ patients satisfied the diagnostic criteria of MS.

The prevalence of double or triple vessel CAD was significantly more common in patients with MS as compared to those without MS (64 vs $40 \%, p=0.02$ ), while the incidence of angiographically normal coronary arteries was also significantly less common in patients with MS as compared to those without MS (8 vs $32 \%$, $\mathrm{p}<0.05)$. Patients with MS had significantly higher mean FRS than those without MS $(16.2$ vs $8.75, \mathrm{p}<0.0001)$. Most patients with MS (78\%) had an intermediate to high 10 year CV risk (>10\%) as estimated by FRS.

We observed that with increasing FRS risk scores, the proportion of patients having MS also increased, of those with low, intermediate and high FRS, 61, 87 and 92\% respectively had Metabolic Syndrome. To observer the independent effects of MS and FRS, patients were categorised into age quartiles ( $<45$ years, $45-$ 55 years, 55-65 years and $>65$ years). Though the incidence of Metabolic Syndrome was nearly similar amongst all age quartiles (75\%, 79\%, 81\%, 82\% respectively), distribution of FRS among different age groups was quite dissimilar. Despite angiographically documented $\mathrm{CAD}$, in patients $<45$ years none of the patients were categorised as high FRS. Only 15 and $17 \%$ patients could be categorised as having high FRS in age groups $45-55$ and 55-65 years, while in patients older than 65 years, $30 \%$ were classified as having high FRS. This analysis helped highlight the obvious limitation of age dependence of FRS.

Conclusions Metabolic syndrome is very commonly observed in Indian patients with angiographically documented CAD; more than $70 \%$ patients with MS have 10 year CV risk of $>10 \%$ as estimated by FRS. Patients with MS had higher incidence of angiographically documented double or triple vessel disease and much less incidence of having normal coronaries as compared to those without MS. In populations like Asian Indians, where patients often develop CAD at a younger age, FRS due to its age dependence may underestimate the CV risk despite the patients having angiographically documented $\mathrm{CAD}$. In our study too, in patients $<45$ years of age, despite presence of angiographic $\mathrm{CAD}$, none of the patients had high FRS. These findings have significant health implications for Asian patients with CAD in whom there should be continued health care emphasis on detection of MS and intensification of adequate preventive strategies. 